\title{
Effect of Yi Guan Jian decoction on differentiation of bone marrow mesenchymalstem cells into hepatocyte-like cells in dimethylnitrosamine-induced liver cirrhosis in mice
}

\author{
YAN XIANG $^{1}$, BING-YAO PANG ${ }^{1}$, YUAN ZHANG ${ }^{1}$, QIAO-LING XIE ${ }^{1}$, \\ YING ZHU ${ }^{1}$, AI-JING LENG ${ }^{2}$, LONG-QING LU ${ }^{1}$ and HAI-LONG CHEN ${ }^{3}$ \\ Departments of ${ }^{1}$ Infectious Diseases, ${ }^{2}$ Pharmacy and ${ }^{3}$ General Surgery, \\ The First Affiliated Hospital of Dalian Medical University, Dalian, Liaoning 116011, P.R. China
}

Received October 18, 2015; Accepted November 8, 2016

DOI: $10.3892 / \mathrm{mmr} .2016 .6083$

\begin{abstract}
Yi Guan Jian decoction (YGD) may induce the differentiation of bone marrow mesenchymal stem cells (BMSCs) into hepatocyte-like cells (HLCs); however, the underlying mechanisms remain to be elucidated. The present study aimed to investigate this process. To do this, a dimethylnitrosamine (DMN)-induced liver cirrhosis mouse model was established. The mice from the model group were randomly divided into three subgroups: i) Negative control, ii) hepatocyte growth factor and iii) YGD. The overall health, liver function and histological alterations were monitored. The expression of $\alpha$-smooth muscle actin ( $\alpha$-SMA), C-X-C chemokine receptor type 4 (CXCR4), extracellular signal-regulated kinase (ERK1/2), nuclear factor $\kappa \mathrm{B}$ p65 subunit $(\mathrm{NF}-\kappa \mathrm{B}$ p65) and $\beta$-catenin were measured by immunohistochemistry, western blotting and reverse transcription-quantitative polymerase chain reaction. Following administration of DMN, the overall health of the mice significantly decreased, with an increase in pathological developments and liver damage resulting in a decrease in liver function. Immunohistochemistry revealed that the expression of $\alpha$-SMA, CXCR4, ERK1/2, NF- $\kappa$ B p65 and $\beta$-catenin was upregulated. Following treatment with YGD, the overall health, liver function and pathology improved. The mRNA and protein expression levels of CXCR4 and ERK1/2 were
\end{abstract}

Correspondence to: Dr Ying Zhu, Department of Infectious Diseases, The First Affiliated Hospital of Dalian Medical University, 222 Zhongshan Road, Dalian, Liaoning 116011, P.R. China E-mail: zhuyingsh52@126.com

Dr Hai-Long Chen, Department of General Surgery, The First Affiliated Hospital of Dalian Medical University, 222 Zhongshan Road, Dalian, Liaoning 116011, P.R. China

E-mail: hailongchen2007@hotmail.com

Key words: Yi Guan Jian decoction, dimethylnitrosamine-induced liver cirrhosis, bone marrow mesenchymal stem cells, differentiation, hepatocyte-like cells upregulated, where as $\alpha$-SMA, NF- $\kappa$ B p65 and $\beta$-catenin levels were downregulated. The results demonstrated that YGD may induce the differentiation of BMSCs into HLCs to reverse DMN-induced liver cirrhosis; this may be achieved via an upregulation of the SDF-1/CXCR4 axis to activate the mitogen activated protein kinase/ERK1/2 signaling pathway.

\section{Introduction}

Bone marrow mesenchymal stem cells (BMSCs) serve as a primary source of adult stem cells for tissue maintenance and regeneration. Bone marrow is the largest reservoir of mesenchymal stem cells (MSCs). A previous study has indicated that BMSCs differentiate into hepatocyte-like cells (HLCs) in vitro and in vivo (1). Liver cirrhosis occurs as a result of the progression of liver fibrosis, when the initial injury is not resolved. Liver fibrosis is a consequence of chronic liver disease characterized by the replacement of liver tissue with an accumulation of collagen and extracellular matrix proteins, scar tissue and regenerative nodules, resulting in dysfunction of the liver. The key to the reversal of liver cirrhosis is the degradation of connective tissue with abnormal hyperplasia, and the regeneration of liver parenchymal cells. In addition, numerous cytokines are important in the development and reversal of liver cirrhosis (2). A previous study has demonstrated that traditional medicines with comprehensive pharmacological effects are beneficial for the treatment of liver cirrhosis (3). Yi Guan Jian decoction (YGD) has been used to treat liver fibrosis and cirrhosis (4). YGD contains Radix Rehmanniae (18 g), Radix Glehniae (9 g), Radix Angelicae Sinensis (9 g), Fructus Lycii (9 g), Radix Ophiopogonis (9 g) and Fructus toosendan (5 g). YGD is a traditional Chinese medicine and has been used to treat hepatic diseases in China for centuries. Our previous study demonstrated that YGD promotes the expression of hepatic oval cells (HOCs) and the differentiation of HOCs into HLCs (5). In the present study, dimethylnitrosamine (DMN) was used to induce liver cirrhosis in mice, and the alterations of liver function and pathology were observed in untreated and YGD-treated groups. In addition, the molecular mechanism underlying the differentiation of mouse BMSCs into HLCs following YGD treatment was identified. 


\section{Materials and methods}

Animals. A total of 192 male and female Kunming mice (age, 4-6 weeks; weight, 16-22 g) were purchased from the Laboratory Animal Center of Dalian Medical University (Dalian, China). The mice were acclimatized to the facility for a week prior to experimentation and maintained under standard conditions (temperature, $20-25^{\circ} \mathrm{C}$; humidity, 60-80\%; 12-h light/dark cycle) with ad libitum water and food. All mice received humane care in accordance with the institutional guidelines. The present study was approved by the Medical Ethics Committee of The First Affiliated Hospital of Dalian Medical University (Dalian, China; approval no. KY2013-49).

Therapeutic agents. YGD includes Radix Rehmanniae (18 g), Radix Glehniae (9 g), Radix Angelicae Sinensis (9 g), Fructus Lycii (9 g), Radix Ophiopogonis (9 g) and Fructus toosendan $(5 \mathrm{~g})$. The formula was decocted in distilled water and prepared in the Pharmacy Department of Dalian Medical University. Hepatocyte growth factor (HGF) was provided by Weihai Sinogen Pharmaceutical Co., Ltd. (Weihai, China) and dissolved in normal saline.

Reagents. DMN was purchased from Tokyo Kasei Kogyo Co., Ltd. (Tokyo, Japan). The primary rabbit anti-mouse polyclonal antibodies against $\alpha$-smooth muscle actin ( $\alpha$-SMA; catalog no. ab66133), C-X-C chemokine receptor type 4 (CXCR4; catalog no. ab2074), extracellular signal-regulated kinase (ERK1/2; catalog no. ab17942) and nuclear factor $\kappa \mathrm{B}(\mathrm{NF}-\kappa \mathrm{B})$ p65 subunit (NF-кBp65; catalog no. ab16502), a rabbit anti-mouse monoclonal antibody against $\beta$-catenin (catalog no. ab32572), a rat anti-mouse monoclonal antibody against cluster of differentiation (CD) 90 (catalog no. ab3105), rabbit anti-rat polyclonal antibodies against albumin (Alb; catalog no. ab135575) and cytokeratin (CK) 18 (catalog no. ab189444), and the secondary antibodies goat anti-rat IgG H\&L (Alexa Fluor $^{\circledR}$ 488; catalog no. ab150157) and goat anti-rabbit IgG H\&L (Alexa Fluor ${ }^{\circledR}$ 647; catalog no. ab150079), were purchased from Abcam (Cambridge, MA, USA). The primary antibody against $\beta$-actin (catalog no. bs-0061R) was obtained from BIOSS (Beijing, China). The secondary antibody peroxidase-conjugated AffiniPure goat anti-rabbit IgG (catalog no. ZB-2301) was obtained from ZSGB-BIO (Beijing, China). The primers, Takara Mini BEST Universal RNA Extraction kit (catalog no. 9767), PrimeScript ${ }^{\mathrm{TM}}$ RT Master mix (catalog no. RR036A) and SYBR ${ }^{\circledR}$ Premix Ex Taq ${ }^{\mathrm{TM}}$ (catalog no. RR420A) were obtained from Takara Bio, Inc. (Otsu, Japan).

Liver cirrhosis model. A liver cirrhosis model was established as previously described by Jenkins et al (6). Animals were randomly divided into two groups. The model group $(\mathrm{n}=172)$ received $10 \mathrm{mg} / \mathrm{kg}$ DMN intraperitoneally once per day for three consecutive days within a week for a total of four weeks. The control group $(n=20)$ mice were injected with an identical volume of normal saline on the same regimen. To monitor the development of liver cirrhosis, five mice from the control group and ten mice from the model group were randomly selected and sacrificed by cervical dislocation, following anesthesia with an intraperitoneal injection of $10 \%$ chloral hydrate (Qingdao Yulong Algae Co., Ltd., Qingdao, China), at weeks 1,2, 3 and 4 following the first injection of DMN.

From the fifth week following the first injection of DMN, DMN administration was ceased, and mice from the model group were further divided into the following three subgroups (n=44/group): i) Negative control (NC), ii) positive control (HGF) and iii) YGD. YGD (10 ml/kg body weight) was administered by oral gavage once daily for four weeks. HGF ( $200 \mu \mathrm{g} / \mathrm{kg}$ body weight) was administered once daily by subcutaneous injection for four weeks. Mice in the NC group received normal saline on the same regimen. A total of 10 mice from each group were randomly selected and sacrificed at weeks 5, 6, 7 and 8 following the first injection of DMN.

Following anesthesia with an intraperitoneal injection of $10 \%$ chloral hydrate, whole blood was collected from mice and the serum was isolated and stored at $-80^{\circ} \mathrm{C}$ for the liver function analysis. The liver was then dissected. A portion of the tissue was partially fixed with formalin and embedded in paraffin for hematoxylin and eosin (HE) staining, Masson's trichrome (MT) staining and immunohistochemistry (IHC). A further portion of the liver tissue was embedded in optimum cutting temperature compound for immunofluorescence, and the remaining tissue was snap-frozen in liquid nitrogen and stored at $-80^{\circ} \mathrm{C}$ for western blotting and reverse transcription-quantitative polymerase chain reaction (RT-qPCR).

Monitoring of mice. Analyses of the overall health of the mice were undertaken, including alterations in weight, feeding, drinking, activity and hair loss.

Measurement of serum liver function. The serum levels of alanine aminotransferase (ALT), aspartate aminotransferase (AST), Alb, total bilirubin (TBil), direct bilirubin (DBil), cholinesterase (CHE), alkaline phosphatase (ALP) and gamma-glutamyltranspeptidase (GGT) were determined using an automatic biochemical analyzer.

Pathology. Macroscopic alterations in the shape, size, color and texture of livers were observed. Paraffin sections were cut into sections (6- $\mu \mathrm{m}$ thick), deparaffinized and rehydrated for HE and MT staining, to enable observation of the alterations to liver cell degeneration, necrosis and the degree of hepatic fibrosis under a light microscope. For HE staining, sections were stained with hematoxylin for $1 \mathrm{~min}$ and washed in tap water, followed by staining with eosin for 1-2 min and further washing in tap water. Sections were subsequently dehydrated, cleared in xylene and mounted. For MT staining, sections were stained with ponceau acid fuchsin solution for $5 \mathrm{~min}$, briefly differentiated with $0.2 \%$ acetic acid aqueous solution, rinsed with distilled water, incubated with $5 \%$ phosphomolybdic acid solution for $8 \mathrm{~min}$ and directly immersed into aniline blue dye for $5 \mathrm{~min}$. Sections were incubated with $0.2 \%$ acetic acid aqueous solution for $2 \mathrm{~min}$ and washed in tap water. Sections were subsequently dehydrated, cleared in xylene and mounted.

$I H C$. Liver sections were deparaffinized and rehydrated, and $5 \%$ bovine serum albumin (BSA; Solarbio, Beijing, China) was used to block non-specific protein binding at room temperature for $2 \mathrm{~h}$. Sections were incubated overnight at $4^{\circ} \mathrm{C}$ with the following primary antibodies: Anti- $\alpha$-SMA (1:200), 
anti-CXCR4 (1:50), anti-ERK1/2 (1:200), anti-NF- $\kappa$ Bp65 (1:500) or anti- $\beta$-catenin (1:500), or $5 \%$ BSA in PBS without antibody as a negative control. The sections were washed and incubated with a peroxidase-conjugated AffiniPure goat anti-rabbit $\mathrm{IgG}$ secondary antibody $(1: 1,000)$ for $30 \mathrm{~min}$ at $37^{\circ} \mathrm{C}$. Color was developed with diaminobenzidine tetrahydrochloride (ZSGB-BIO). Slides were counterstained with hematoxylin, mounted and visualized under a light microscope.

Immunofluorescence. Following blocking as aforementioned, slides were incubated with anti-CD90 (1:300) and anti-Alb (1:100) or anti-CK18 (1:100) overnight at $4^{\circ} \mathrm{C}$. Goat anti-rat IgG-AlexaFluor488 (1:200) and goat anti-rabbit IgG-AlexaFluor647 (1:200) secondary antibodies were added and incubated in the dark for $1 \mathrm{~h}$ at room temperature. Images were captured by confocal laser scanning microscopy (Leica Microsystems, GmbH, Wetzlar, Germany). Identical exposure times and light intensities were applied to all images. ImageJ software was used (version, JAVA 1.6.0-24 for Windows; National Institutes of Health, Bethesda, MD, USA).

Western blotting. The liver tissues were homogenized in radioimmunoprecipitation assay buffer containing a protease inhibitor cocktail (Beyotime Institute of Biotechnology, Haimen, China). Total protein $(25 \mu \mathrm{g})$ was loaded into each well, subjected to $10 \%$ SDS-PAGE and transferred onto polyvinylidene difluoride membranes. Following this, membranes were blocked in 5\% non-fat milk in PBS containing Tween 20 at $37^{\circ} \mathrm{C}$ for $1 \mathrm{~h}$, and subsequently incubated with antibodies against $\alpha$-SMA (1:500), CXCR4 (1:1,000), ERK1/2 $(1: 1,000), \mathrm{NF}-\kappa \mathrm{Bp} 65(1: 1,000), \beta$-catenin $(1: 5,000)$ or $\beta$-actin $(1: 1,500)$, which served as an internal standard, overnight at $4^{\circ} \mathrm{C}$. Membranes were incubated with a peroxidase-conjugated AffiniPure goat anti-rabbit IgG secondary antibody (dilution, $1: 1,000$ ) at $37^{\circ} \mathrm{C}$ for $1 \mathrm{~h}$. Proteins were visualized with a Diaminobenzidine Horseradish Peroxidase Color Development kit (Beyotime Institute of Biotechnology) and quantified by densitometry using the AlphaView Stand Alone Analysis software (ProteinSimple, version 3.4.0.0; San Jose, CA, USA).

$R T-q P C R$. Total RNA was purified from liver tissues using a Takara MiniBEST Universal RNA Extraction kit, and subjected to RT using PrimeScript RT Master mix. qPCR was performed using SYBR Premix Ex Taq and aStepOnePlus ${ }^{\mathrm{TM}}$ Real-Time PCR system (Applied Biosystems; Thermo Fisher Scientific, Inc., Waltham, MA, USA). GAPDH served as an internal control. Sequences of primers were as follows: Forward, 5'-GACAATGGCTCTGGGCTCTGTA-3' and reverse, 5'-TTT GGCCCATTCCAACCATTA-3' for $\alpha$-SMA; forward, 5'-CCA TGGAACCGATCAGTGTG-3' and reverse, 5'-GCCGACTAT GCCAGTCAAGAA-3' for CXCR4; forward, 5'-CACACG TTGGTACAGAGCTCCAG-3' and reverse, 5'-TGCAGCCCA CAGACCAAATATC-3' for ERK1; forward, 5'-CTGGAC CAGCTCAACCACATTC-3' and reverse, 5'-AGGTAGTTT CGGGCCTTCATGTTA-3' for ERK2; forward, 5'- AGCACA GATACCACCAAGACACA-3' and reverse, 5'-CAGGTCTCG CTTCTTCACACAC-3' for NF- $\mathrm{Bp} 65$; forward, 5'-GCCACA GGATTACAAGAAGC-3' and reverse, 5'-CCACCAGAGTGA AAAGAACG-3' for $\beta$-catenin; and forward, 5'-AAATGG
TGAAGGTCGGTGTGAAC-3' and reverse, 5'-CAACAA TCTCCACTTTGCCACTG-3' for GAPDH. The details of the thermocycling conditions were as follows: $95^{\circ} \mathrm{C}$ for $30 \mathrm{sec}$ (initial denaturation), followed by 40 cycles at $95^{\circ} \mathrm{C}$ for $5 \mathrm{sec}$ (exact denaturation) and $60^{\circ} \mathrm{C}$ for $30 \mathrm{sec}$ (primer annealing and PCR product elongation). The relative quantity of target genes mRNA was normalized to that of GAPDH using the $2^{-\Delta \Delta C q}$ method, as previously described (7).

Statistical analysis. Statistical analysis was performed using SPSS software version 19.0 (IBM SPSS, Armonk, NY, USA) and data are presented as the mean \pm standard deviation. Data were analyzed using Shapiro-Wilk (normality) and Levene's (homogeneity) tests. Differences between the control and treated groups were analyzed using one-way analysis of variance, followed by Tukey's post hoc test. $\mathrm{P}<0.05$ was considered to indicate a statistically significant difference.

\section{Results}

Physical state of mice. Immediately following administration of DMN, the model group exhibited anxiety and were irritable, gradually becoming inactive and calm over a period of $5 \mathrm{~h}$. Behavioral improvement was observed 4 days later, and returned to normal 7 days later, except with hair loss and a small quantity of ascites. There were no significant differences in eating, drinking and weight between groups of mice at any time point. The symptoms improved following treatment with YGD or HGF.

Liver function decreases in the model group. ALT, AST, TBil, DBil and ALP levels at 1-4 weeks and GGT levels at 1-3 weeks increased $(\mathrm{P}<0.05)$, and Alb levels at $1-4$ weeks and CHE levels at 2-4 weeks decreased $(\mathrm{P}<0.05)$, in the model compared with the control group. These results revealed that liver function gradually decreased in the model group (Table I).

Serum liver function improves in the HGF and YGD groups. Following HGF treatment, ALT and AST levels at 1-4 weeks decreased $(\mathrm{P}<0.05)$, Alb levels at 1-4 weeks and $\mathrm{CHE}$ levels at 3-4 weeks increased $(\mathrm{P}<0.05)$, DBil levels decreased at 4 weeks $(\mathrm{P}<0.05)$, as did TBil levels at $3-4$ weeks $(\mathrm{P}<0.05)$ and ALP levels at 1, 3 and 4 weeks $(\mathrm{P}<0.05)$, compared with the model group. Therefore, improvement of liver function was observed in the HGF group. Compared with the NC group at the same time point, the levels of ALT, AST and GGT at 1-4 weeks decreased $(\mathrm{P}<0.05)$, Alb levels increased at 1-4 weeks $(\mathrm{P}<0.05)$, TBil and ALP levels decreased at week 4 $(\mathrm{P}<0.05)$, DBil levels decreased at weeks 1 and $4(\mathrm{P}<0.05)$, and CHE levels increased at weeks 1,3 and 4 ( $\mathrm{P}<0.05$; Table II).

Following YGD treatment, ALT and AST levels at 1-4 weeks decreased $(\mathrm{P}<0.05)$, Alb and CHE levels at 1-4 weeks increased $(\mathrm{P}<0.05)$, TBil and GGT levels decreased at 4 weeks $(\mathrm{P}<0.05)$, as did DBil levels at $2-4$ weeks $(\mathrm{P}<0.05)$ and ALP levels at 1,2 and 4 weeks $(\mathrm{P}<0.05)$, compared with the model group at 4 weeks. Compared with the NC group at the same time point, the levels of ALT and AST at 1-4 weeks decreased $(\mathrm{P}<0.05)$, Alb and CHE levels increased at $1-4$ weeks $(\mathrm{P}<0.05)$, and the levels of TBil at week 4, DBil at weeks 1, 3 and 4, ALP at weeks 1, 2 and 4, and GGT at week 1, increased $(\mathrm{P}<0.05)$. 


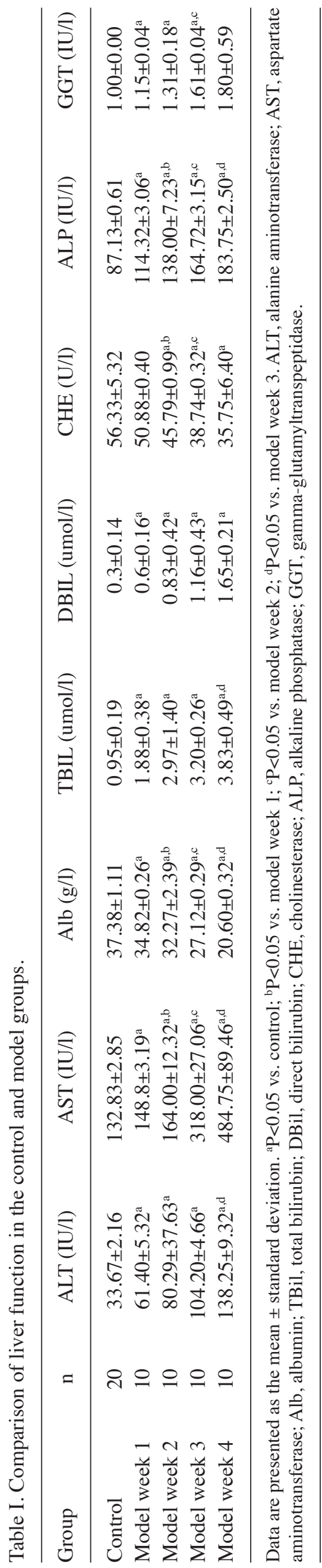

The results suggested that liver function improved gradually in the YGD group (Table II).

Additionally, CHE levels were increased $(\mathrm{P}<0.05)$ following YGD treatment compared with the HGF group at week 1. AST and GGT levels were decreased $(\mathrm{P}<0.05)$ in the YGD group compared with the HGF group at week 2. ALT, AST and GGT levels decreased $(\mathrm{P}<0.05)$, whereas CHE levels increased, in the YGD group compared with the HGF group at week 3. AST, ALP and GGT levels decreased $(\mathrm{P}<0.05)$ in the YGD group compared with the HGF group at 4 weeks. There were no significant differences $(\mathrm{P}>0.05)$ between the two groups except for those described above (Table II).

Liver tissue alterations. The control group liver presented a reddish brown color, with a smooth and soft surface. In the model group, the liver tissues were markedly swollen and green/yellow, with part of the liver surface nodular, hard and with a blunt edge. In the YGD group, the liver swelling decreased and the liver surface was yellow/brown and smooth. In the HGF group, the liver tissue appeared similar to the YGD group; however, alterations in this group were less marked compared with the YGD group.

Liver HE staining. Model group liver cells at 1 week were markedly swollen and degenerated, exhibiting a visible infiltration of inflammatory cells in the necrotic area. At weeks 2 and 3 , inflammatory cell infiltration had increased, with destruction of hepatic lobules and portal area structure, and disordered arrangement of liver cells. At 4 weeks liver cells exhibited increased degeneration, steatosis around portal areas and centric veins, with collagenous fibers in the portal area.

In the YGD group at weeks 1-3, the degeneration necrosis of liver cells gradually reduced, with disordered arrangement of the regenerating hepatic cord, dual-core liver cells were visible under a high power lens and steatosis around portal areas and centric veins gradually reduced. At week 4 , the liver cells almost resembled those in the control group. Similar hepatic pathology and ultrastructure alterations were observed in the HGF group. In the NC group at weeks 1-4, the degeneration necrosis, steatosis around portal areas and centric veins, with collagenous fibers in the portal area of liver cells were continued. A small quantity of liver cell regeneration could be seen at week 4 (Fig. 1A).

Liver MT staining. In the model group, fibrous septa formed in the portal areas of livers at week 1, extended and subsequently spread to the liver parenchyma. The fibrous septa thickened at weeks 2 and 3 and extended to the intralobular area surrounding the liver cells, with the central vein wall thickened. The hyperplasia of fibrous tissue and pseudolobule proliferation occurred in mice liver tissues at week 4 , in which atresia, absence, bias and double central vein structure were visible. These results suggested that the liver cirrhosis model had been successfully established.

In the YGD group the hyperplasia of fibrous tissue and fibrous septa gradually reduced at weeks 1-3. The collagen fiber levels resembled those in the control group by week 4, and a small quantity of collagen fibers were viewed only near the portal area and central vein. In the HGF group, there was a decrease in fibrous septa and collagen fiber levels. In the NC 
A
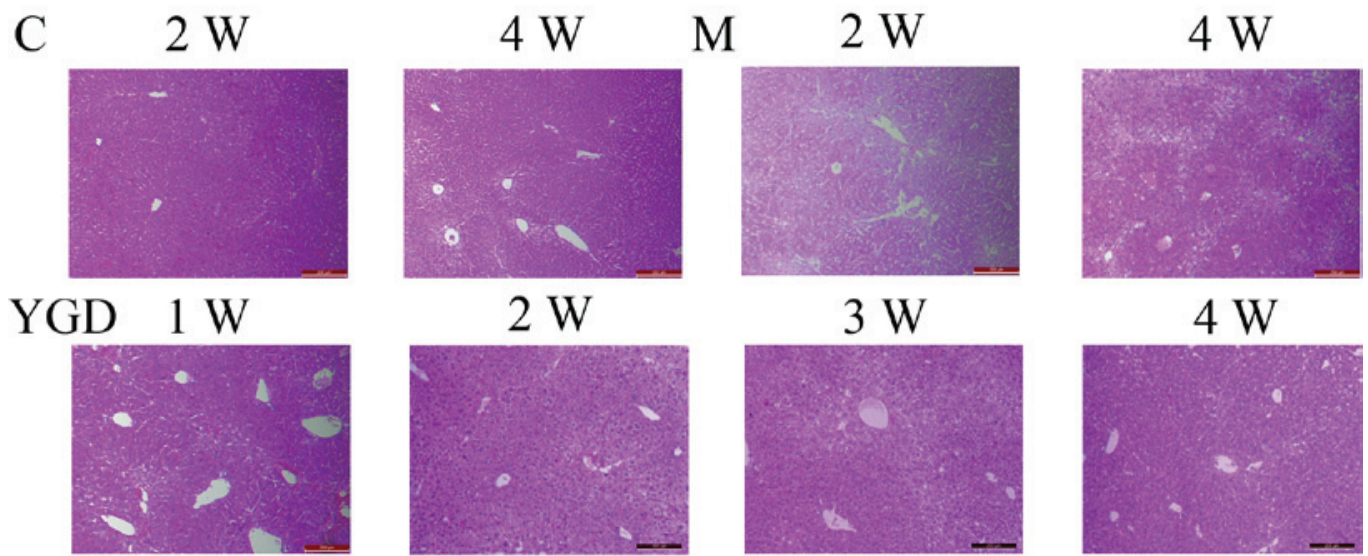

$2 \mathrm{~W}$

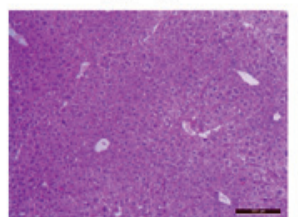

$3 \mathrm{~W}$

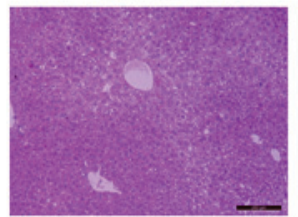

$4 \mathrm{~W}$

HGF $1 \mathrm{~W}$

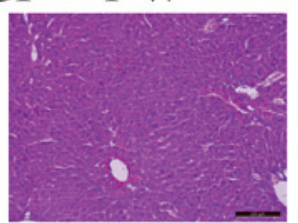

$2 \mathrm{~W}$

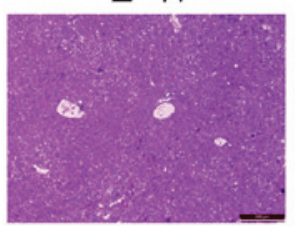

$3 \mathrm{~W}$
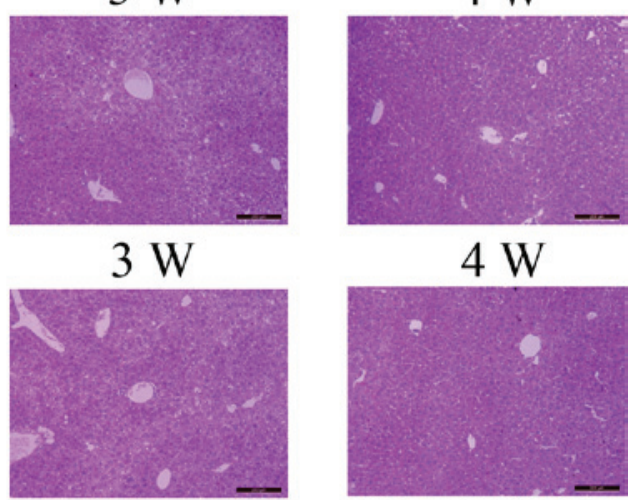

$4 \mathrm{~W}$

$\mathrm{NC} \quad 1 \mathrm{~W}$

$2 \mathrm{~W}$
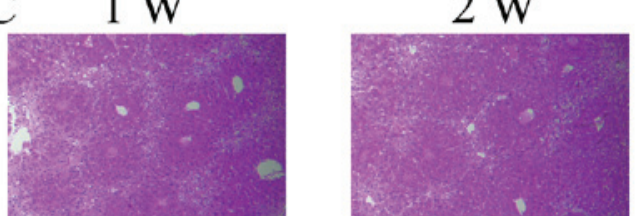

$3 \mathrm{~W}$
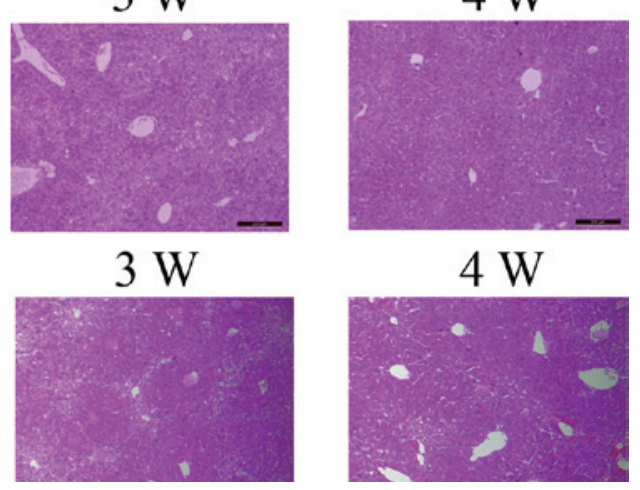

B

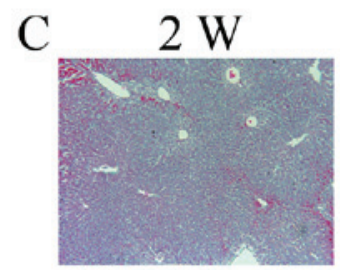

YGD $1 \mathrm{~W}$
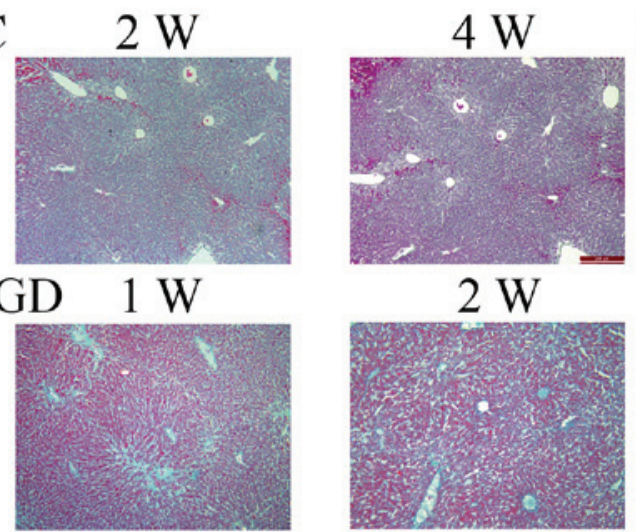

M

$2 \mathrm{~W}$

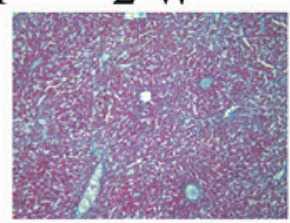

$2 \mathrm{~W}$

$3 \mathrm{~W}$
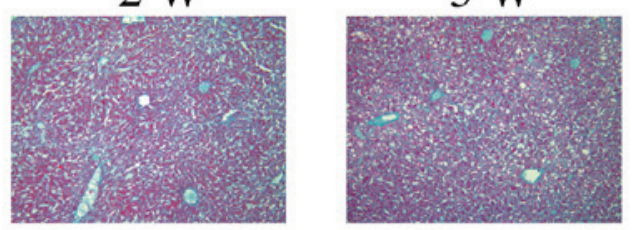

$3 \mathrm{~W}$

HGF $1 \mathrm{~W}$

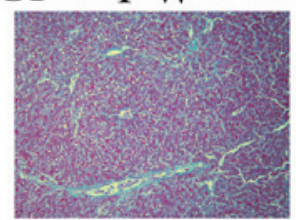

$2 \mathrm{~W}$
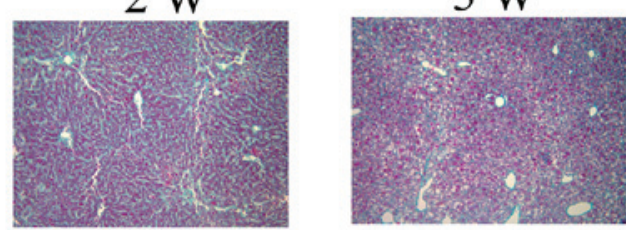

$2 \mathrm{~W}$

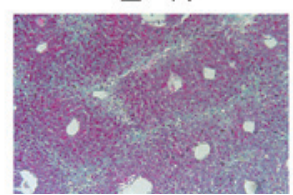

$3 \mathrm{~W}$
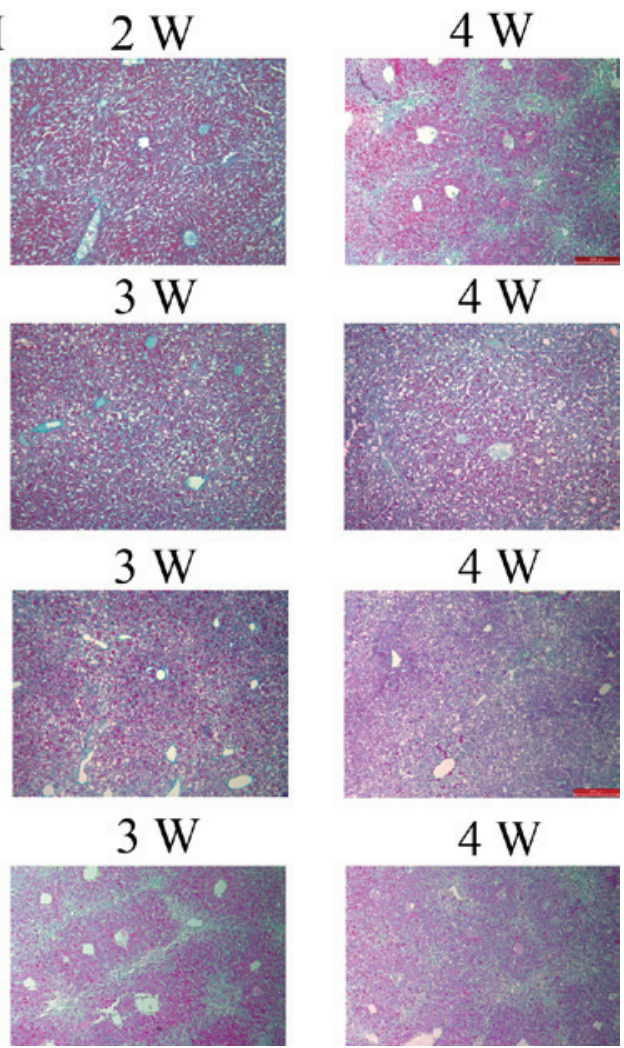

$4 \mathrm{~W}$

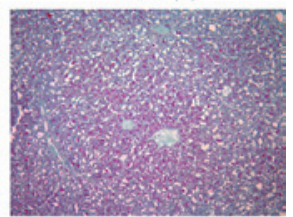

$4 \mathrm{~W}$

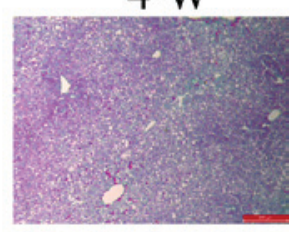

$4 \mathrm{~W}$

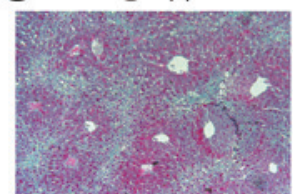

Figure 1. Comparison of liver histology in mice with DMN-induced liver cirrhosis. Mice were injected with DMN or normal saline for 4 weeks. Following 4 weeks of DMN treatment, mice were subsequently treated with YGD or HGF for a further 4 weeks. Liver tissues were stained with (A) hematoxylin and eosin and (B) Masson's trichrome. Liver damage induced by DMN was alleviated by YGD or HGF treatment. Magnification, x100; scale bar=200 $\mu \mathrm{m}$. YGD, Yi Guan Jian decoction; HGF, hepatocyte growth factor; DMN, dimethylnitrosamine; C, control; M, model; NC, negative control; W, week. 
A

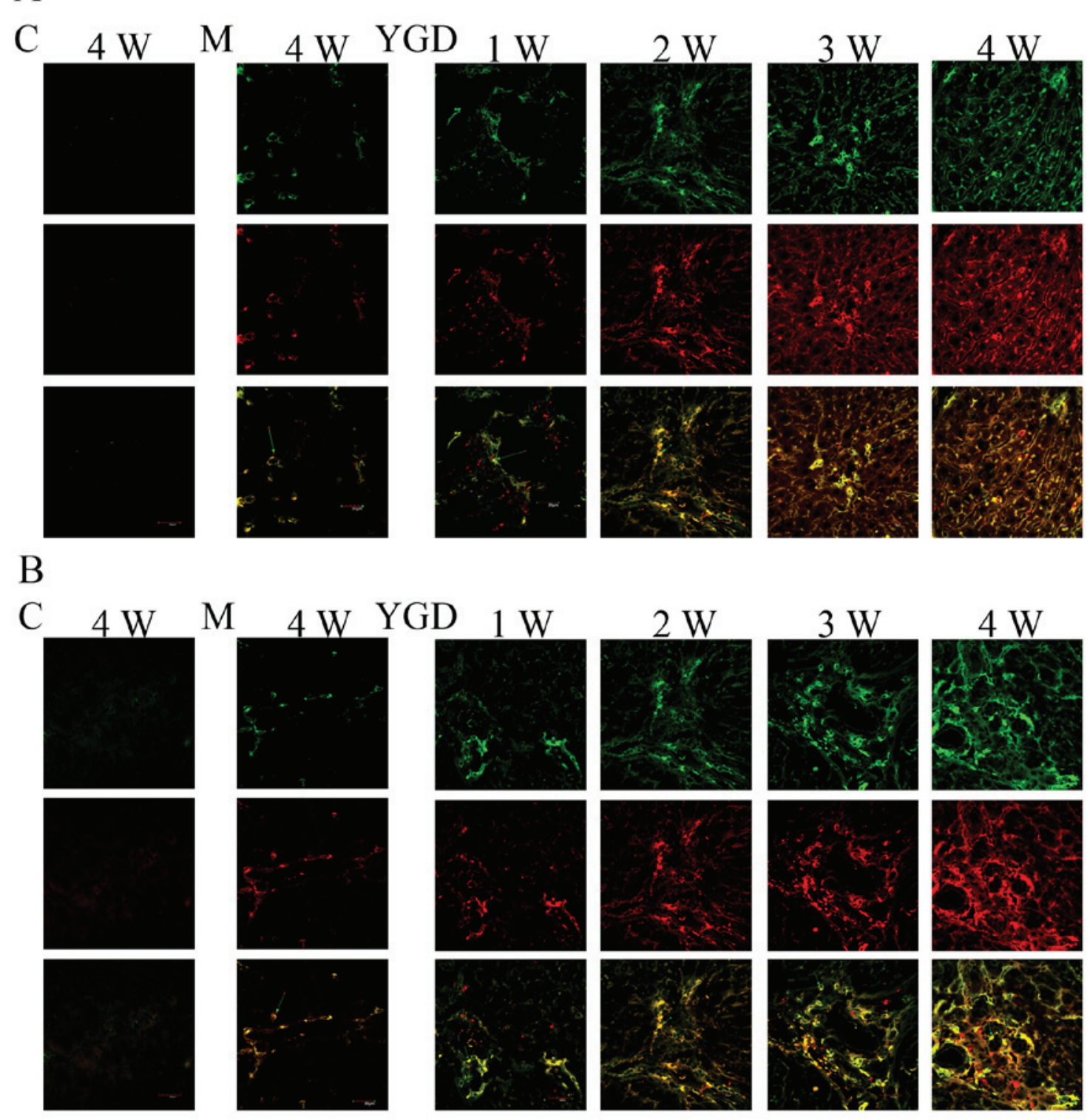

Figure 2. DMN and YGD regulate the differentiation of BMSCs into hepatocytes and biliary epithelial cells. Mice were injected with DMN or normal saline for 4 weeks. Following 4 weeks of DMN treatment, mice were treated with YGD or HGF for a further 4 weeks. (A) The expression of CD90 (green, top row) and Alb (red, middle row) was detected by immunofluorescence, with the co-localization analyzed by merging these images (bottom row). (B) The expression of CD90 (green, top row) and CK18 (red, middle row) was detected by immunofluorescence, with the co-localization analyzed by merging these images (bottom row). Expression and co-localization was increased by DMN injection and further increased by YGD treatment. Magnification, x60. YGD, Yi Guan Jian decoction; HGF, hepatocyte growth factor; DMN, dimethylnitrosamine; CD, cluster of differentiation; Alb, Albumin; CK18, cytokeratin 18; C, control; M, model; W, week.

group at weeks 1-4, pseudolobules, atresia, absence, bias and double central vein structure remained visible (Fig. 1B).

Co-localization of CD90 with Alb or CK18. To monitor BMSCs and their potential to differentiate into hepatocytes as well as biliary epithelial cells during the development and reversal of liver cirrhosis, double labeling immunofluorescence was performed in liver tissue from the model and YGD groups. The expression of the BMSC marker CD90 with the hepatocyte marker Alb or the biliary epithelial marker CK18 was investigated. In the model group, there was minimal co-localization between CD90 and Alb or CK18 at weeks 1 and 2, while at weeks 3 and 4 the co-localization gradually increased. The co-localization of CD90 with Alb or CK18 increased markedly following YGD treatment, and reached a peak at week 4 (Fig. 2).

Protein expression of $\alpha-S M A, C X C R 4, E R K 1 / 2, N F-\kappa B p 65$ and $\beta$-catenin. IHC on the control group revealed no or very few positively stained cells. In the model group, $\alpha$-SMA, CXCR4, ERK1/2, NF- $\kappa$ B p65 and $\beta$-catenin staining markedly increased and was primarily concentrated in the portal area, near the fibrous septa and hepatic cord. Following YGD or HGF administration, CXCR4 and ERK1/2 staining gradually increased compared with the $\mathrm{NC}$ group at the same point and the model group at week 4, peaking at 4 weeks in the YGD and HGF groups. $\alpha$-SMA, NF- $\kappa$ B p 65 and $\beta$-catenin levels gradually decreased the $\mathrm{NC}$ group at the same point 

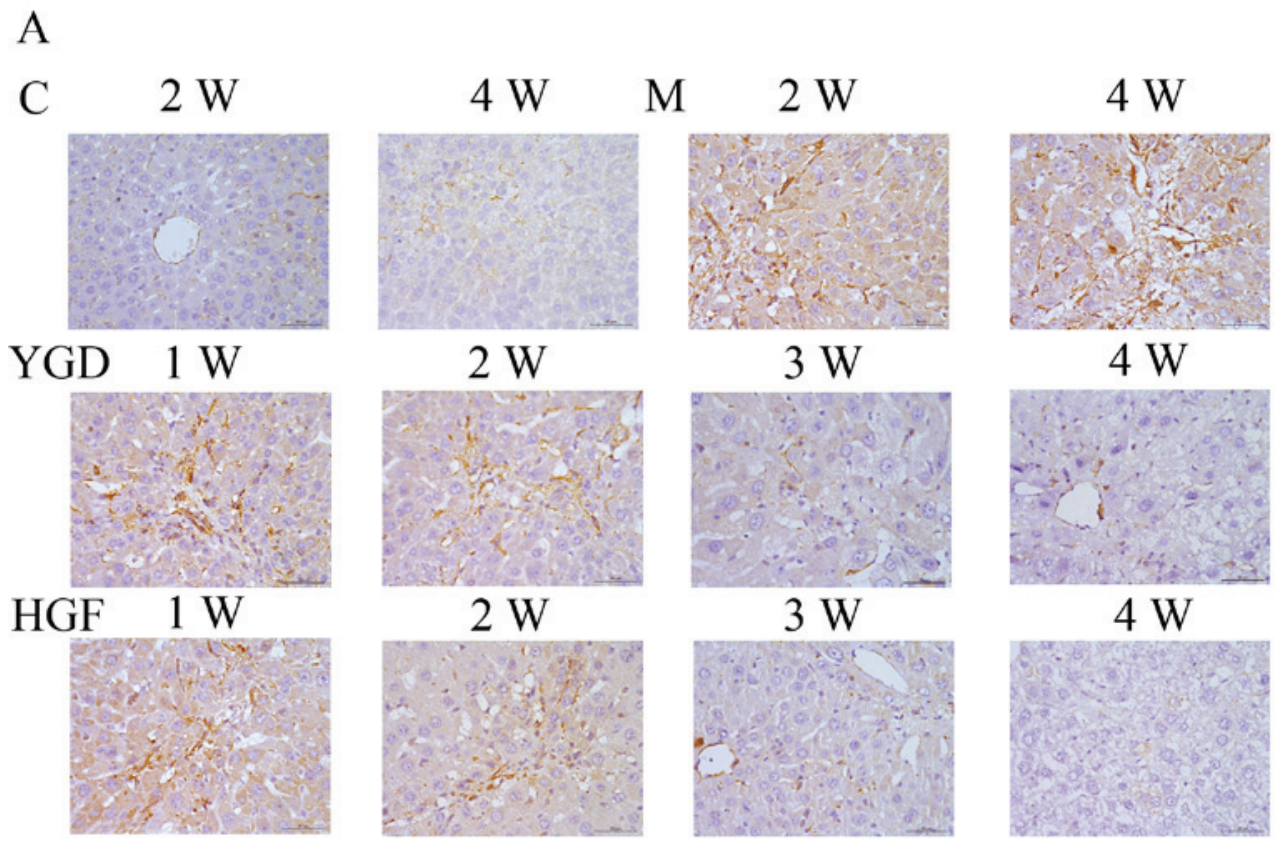

\section{$2 \mathrm{~W}$}

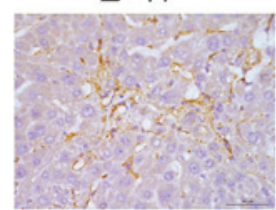

$2 \mathrm{~W}$

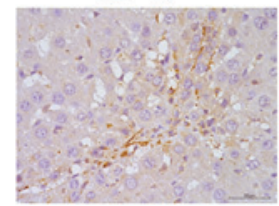

$2 \mathrm{~W}$
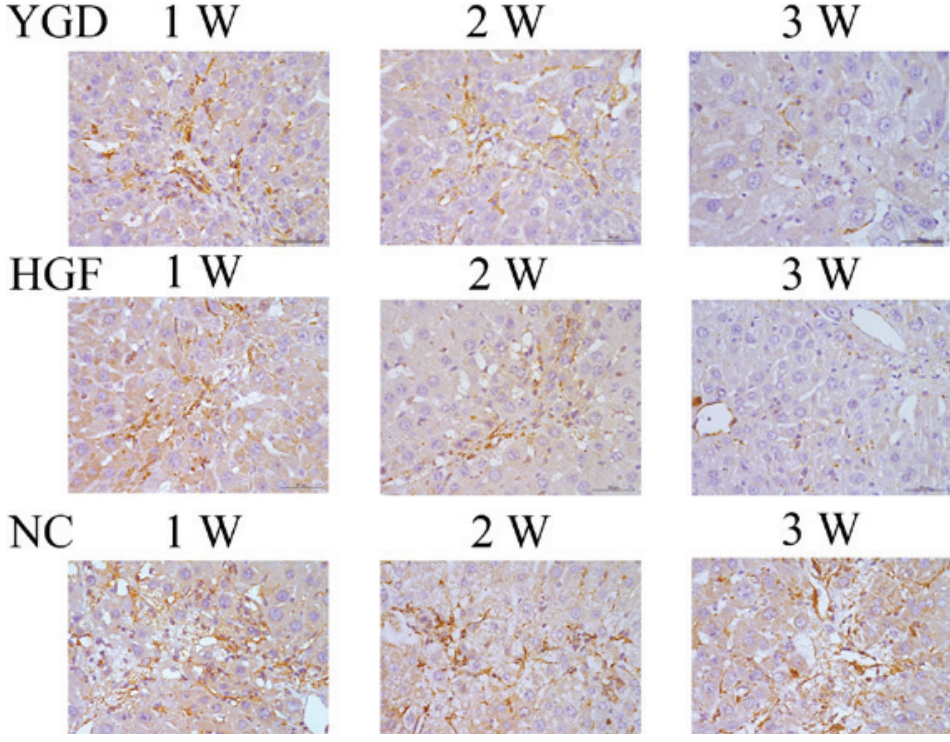

$3 \mathrm{~W}$

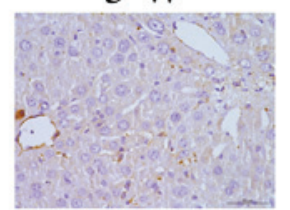

$3 \mathrm{~W}$

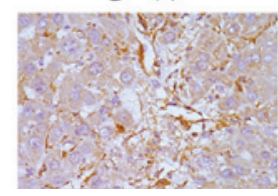

$4 \mathrm{~W}$

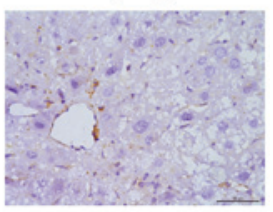

$4 \mathrm{~W}$

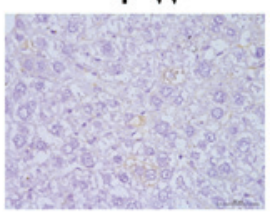

$4 \mathrm{~W}$

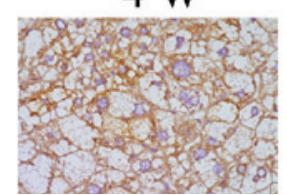

B

C $2 \mathrm{~W}$

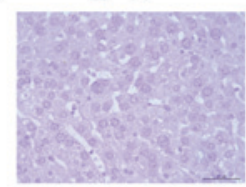

YGD $1 \mathrm{~W}$

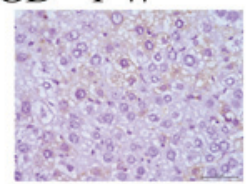

HGF $1 \mathrm{~W}$

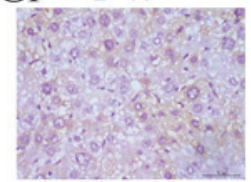

$\mathrm{NC}$

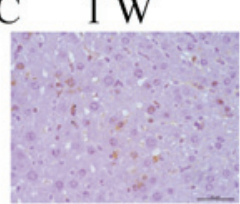

$4 \mathrm{~W}$

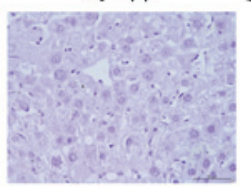

$2 \mathrm{~W}$

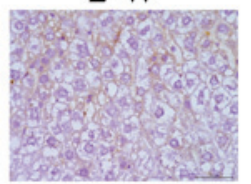

$2 \mathrm{~W}$

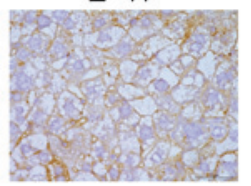

$2 \mathrm{~W}$

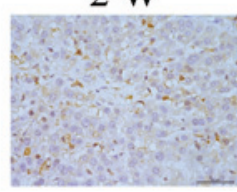

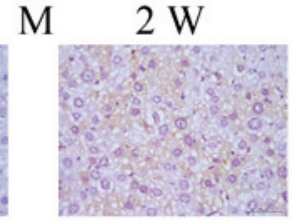

$3 \mathrm{~W}$

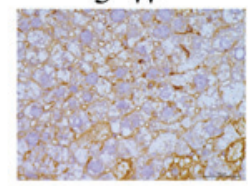

$3 \mathrm{~W}$

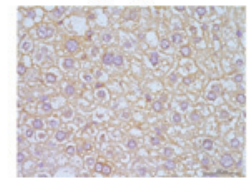

$3 \mathrm{~W}$

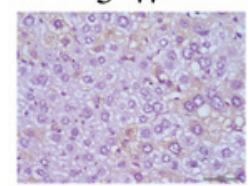

$4 \mathrm{~W}$

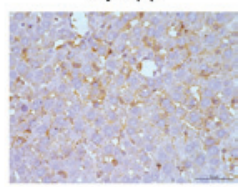

$4 \mathrm{~W}$

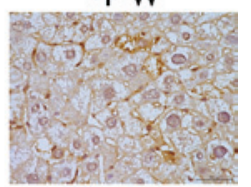

$4 \mathrm{~W}$

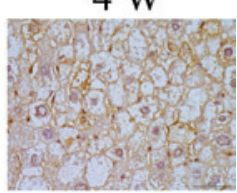

$4 \mathrm{~W}$

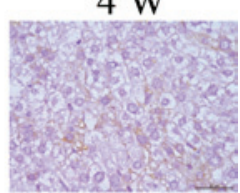

Figure 3. DMN, YGD and HGF regulate the expression of $\alpha$-SMA, CXCR4, ERK1/2, NF-kB p65 and $\beta$-catenin. Mice were injected with DMN or normal saline for 4 weeks. Following 4 weeks of DMN treatment, mice were treated with YGD or HGF for a further 4 weeks. Liver sections were stained for (A) $\alpha$-SMA (B) CXCR4.

and compared with the model group at week 4; by week 4 in the YGD and HGF groups, the expression was almost at the level of the control group (Fig. 3). As the model group week 4 demonstrated more severe damage, according to measurements 


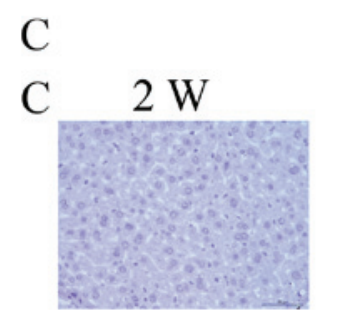

YGD $1 \mathrm{~W}$

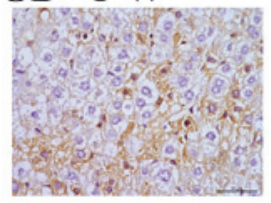

HGF $1 \mathrm{~W}$

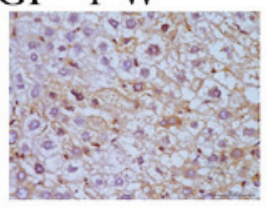

$\mathrm{NC} \quad 1 \mathrm{~W}$

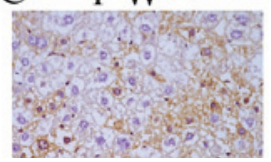

$\mathrm{D}$
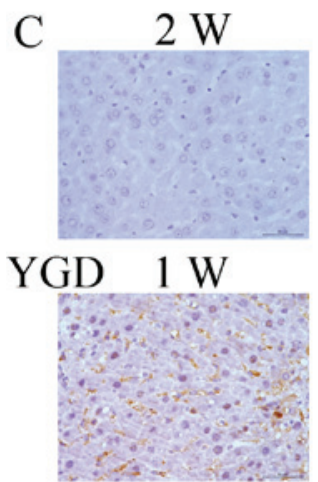

HGF $1 \mathrm{~W}$

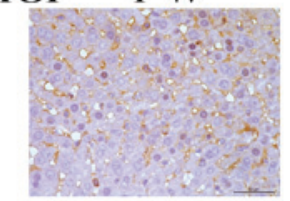

NC

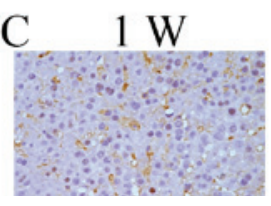

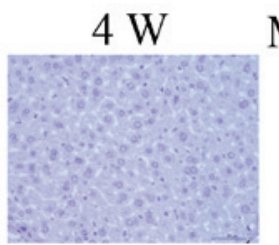

$2 \mathrm{~W}$

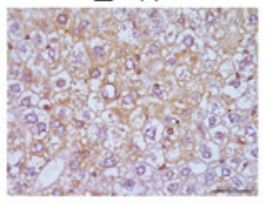

$2 \mathrm{~W}$

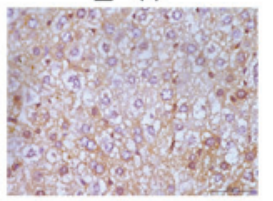

$2 \mathrm{~W}$

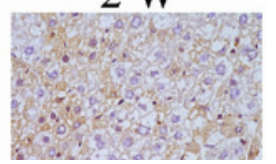

$4 \mathrm{~W}$

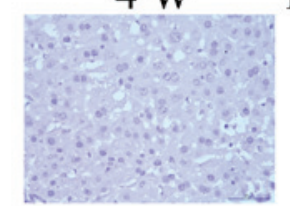

$2 \mathrm{~W}$

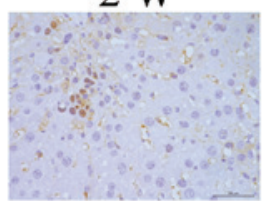

$2 \mathrm{~W}$

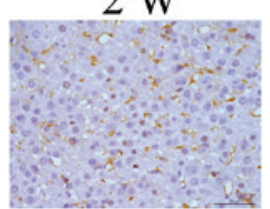

$2 \mathrm{~W}$

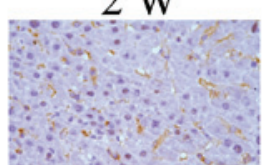

M

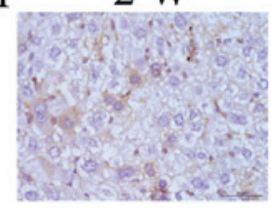

$3 \mathrm{~W}$

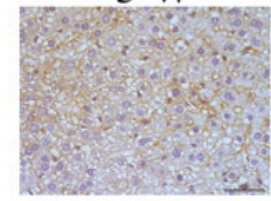

$3 \mathrm{~W}$

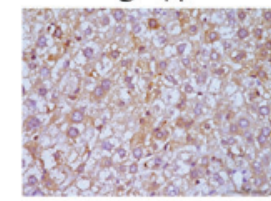

$3 \mathrm{~W}$
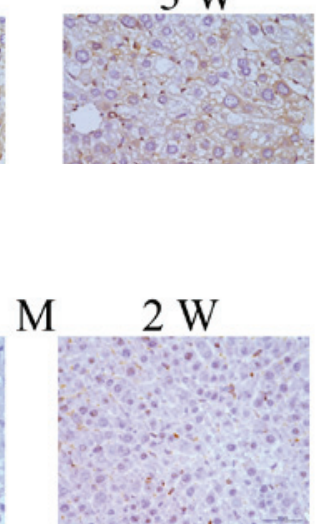

$3 \mathrm{~W}$

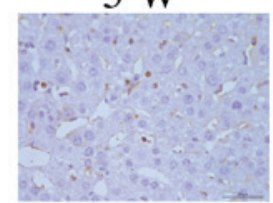

$3 \mathrm{~W}$

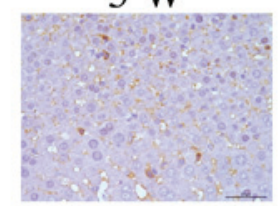

$3 \mathrm{~W}$

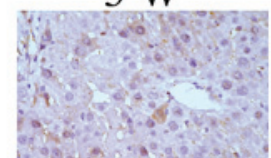

$4 \mathrm{~W}$

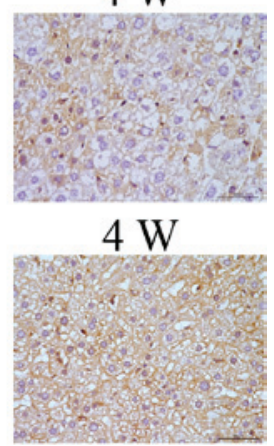

$4 \mathrm{~W}$

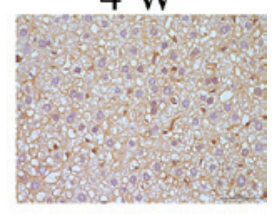

$4 \mathrm{~W}$
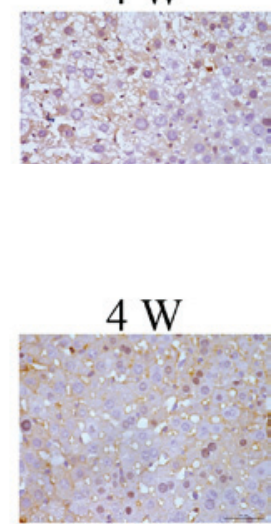

$4 \mathrm{~W}$

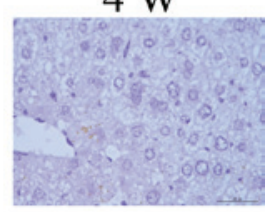

$4 \mathrm{~W}$

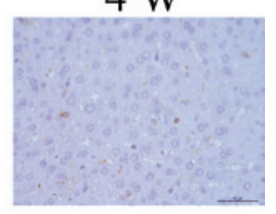

$4 \mathrm{~W}$

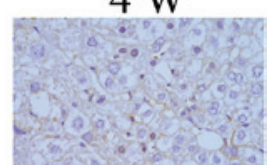

Figure 3. Continued. (C) ERK1/2 (D) NF-кB p65.

of serum liver function, pathology and IHC, compared with the NC group at any time point, it was selected for comparison with the YGD and HDF groups in subsequent experiments.

Western blotting revealed that the protein expression levels of $\alpha$-SMA, ERK1/2, NF- $\kappa$ B p65 and $\beta$-catenin in the model group at 4 weeks were increased compared with the control group $(\mathrm{P}<0.05)$. Following YGD treatment, the CXCR4 and ERK1/2 protein expression levels significantly increased $(\mathrm{P}<0.05)$ compared with the model group at week 4 , with a peak at 4 weeks. $\alpha$-SMA, NF- $\mathrm{B}$ p65 and $\beta$-catenin protein expression levels significantly decreased following YGD treatment in a time-dependent manner $(\mathrm{P}<0.05)$ compared 
E
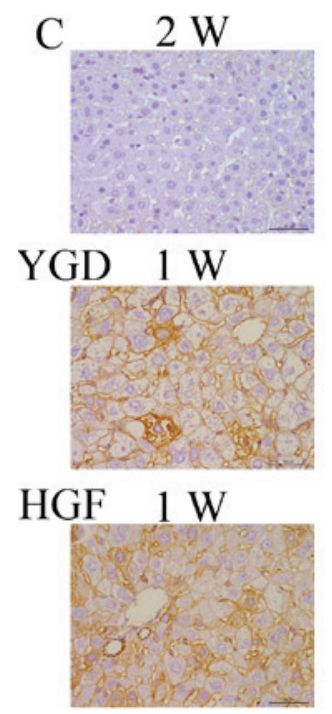

$\mathrm{NC}$

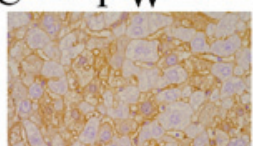

$4 \mathrm{~W}$

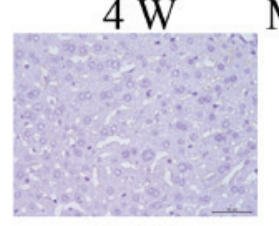

$2 \mathrm{~W}$

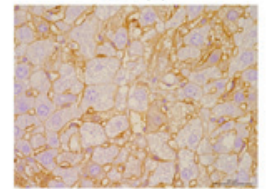

$2 \mathrm{~W}$

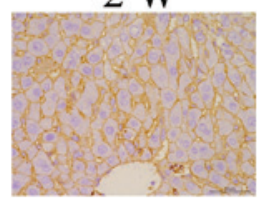

$2 \mathrm{~W}$
M

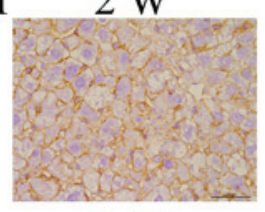

$3 \mathrm{~W}$

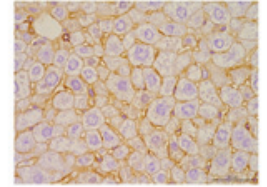

$3 \mathrm{~W}$

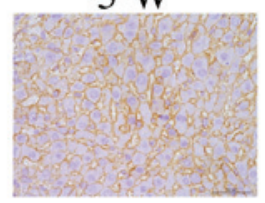

$3 \mathrm{~W}$

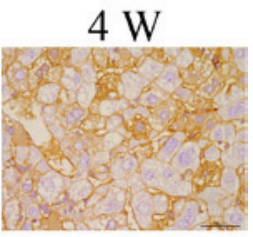

$4 \mathrm{~W}$

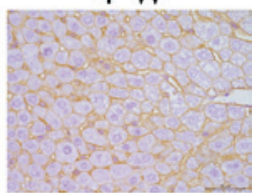

$4 \mathrm{~W}$

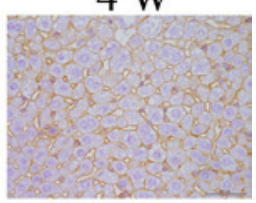

$4 \mathrm{~W}$
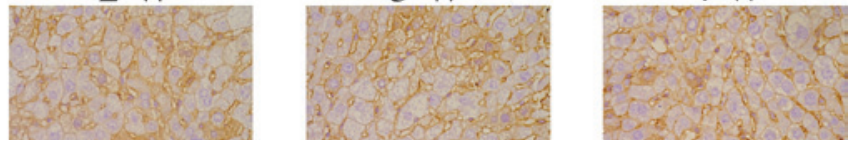

Figure 3. Continued. (E) $\beta$-catenin. A representative image from each group is presented. Very little staining was observed in the control group. YGD or HGF treatment increased CXCR4 and ERK1/2 staining, and decreased $\alpha$-SMA, NF- $\mathrm{kB}$ p 65 and $\beta$-catenin staining, compared with the DMN-injected group. Magnification, x400; scale bar=50 $\mu \mathrm{m}$.YGD, Yi Guan Jian decoction; HGF, hepatocyte growth factor; DMN, dimethylnitrosamine; $\alpha$-SMA, $\alpha$-smooth muscle actin; CXCR4, C-X-C chemokine receptor type 4; ERK1/2, extracellular signal-regulated kinase 1/2; NF- $\mathrm{B}$ p65, nuclear factor $\kappa \mathrm{B}$ p65 subunit; C, control; $\mathrm{M}$, model; $\mathrm{NC}$, negative control; $\mathrm{W}$, week.

A

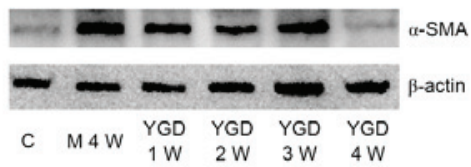

B

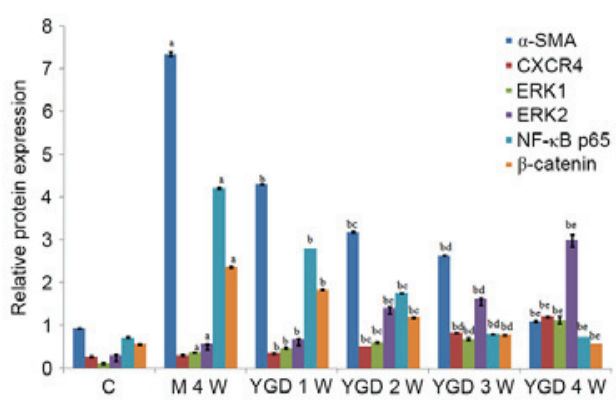

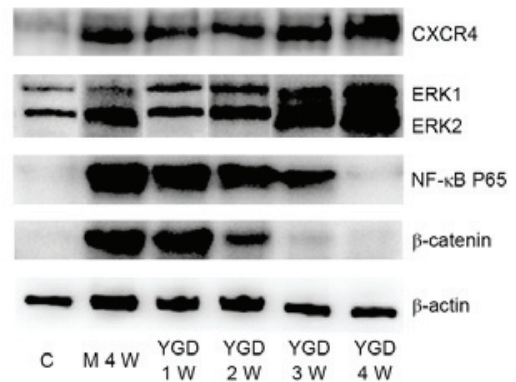

C

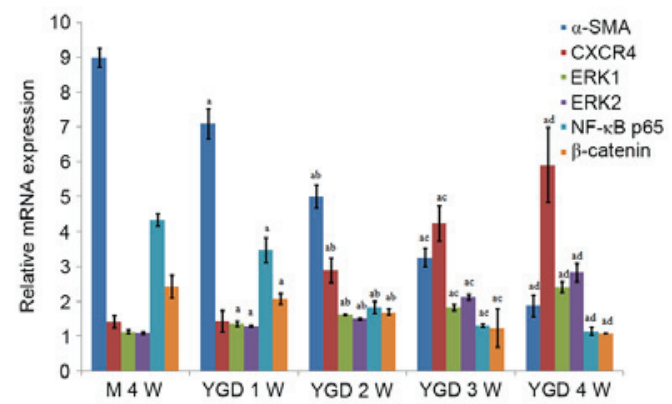

Figure 4. DMN and YGD regulate mRNA and protein expression levels of $\alpha$-SMA, CXCR4, ERK1/2, NF-kB p65 and $\beta$-catenin. Mice were injected with DMN or normal saline for 4 weeks. Following 4 weeks of DMN treatment, mice were treated with YGD or HGF for a further 4 weeks. (A) The protein expression levels of $\alpha$-SMA, CXCR4, ERK1/2, NF- $\mathrm{kB}$ p 65 and $\beta$-catenin were detected by western blotting and (B) the western blots were quantified. ${ }^{a} \mathrm{P}<0.05$ vs. control; ${ }^{\mathrm{b}} \mathrm{P}<0.05$ vs. model week 4 ; ${ }^{\mathrm{C}} \mathrm{P}<0.05$ vs. YGD week 1 ; ${ }^{\mathrm{d}} \mathrm{P}<0.05$ vs. YGD week 2 ; and ${ }^{\mathrm{e}} \mathrm{P}<0.05$ vs. YGD week 3 . (C) Reverse transcription-quantitative polymerase chain reaction was performed to analyze the mRNA expression levels. ${ }^{\mathrm{P}} \mathrm{P}<0.05 \mathrm{vs}$. model week 4 ; ${ }^{b} \mathrm{P}<0.05 \mathrm{vs}$. YGD week 1 ; ${ }^{\mathrm{C}} \mathrm{P}<0.05 \mathrm{vs}$. YGD week 2 ; and ${ }^{d} \mathrm{P}<0.05$ vs. YGD week 3. YGD, Yi Guan Jian decoction; DMN, dimethylnitrosamine; $\alpha$-SMA, $\alpha$-smooth muscle actin; CXCR4, C-X-C chemokine receptor type 4; ERK1/2, extracellular signal-regulated kinase 1/2; NF-кB p65, nuclear factor $\kappa \mathrm{B}$ p65 subunit; C, control; M, model; W, week. 


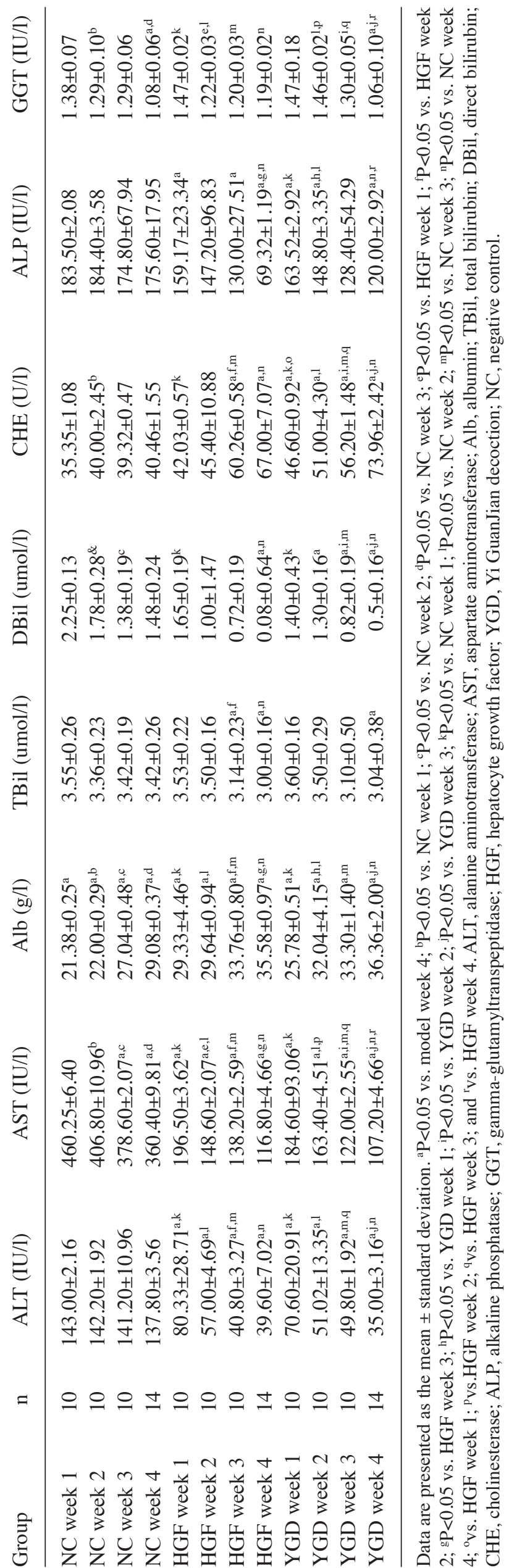


with the model group at week 4 , almost reaching control group levels ( $\mathrm{P}<0.05$; Fig. 4A and B; Table III).

mRNA expression levels of $\alpha-S M A, C X C R 4, E R K 1 / 2, N F-\kappa B$ p65 and $\beta$-catenin. In the model group at week 4 , the mRNA relative expression levels were as follows: $\alpha$-SMA, 8.998 \pm 0.267 ; CXCR4, 1.424 \pm 0.186 ; ERK1, 1.133 \pm 0.050 ; ERK2, 1.104 \pm 0.046 ; NF-кB p65, 4.338 \pm 0.1761 ; and $\beta$-catenin, $2.433 \pm 0.324$.

These values were all greater compared with the control group. Following YGD treatment, the relative mRNA expression levels of CXCR4 and ERK1/2 gradually increased, with maximum levels achieved at week 4 . The relative mRNA expression levels of CXCR4 at 2-4 weeks and ERK1/2 at 1-4 weeks all significantly increased $(\mathrm{P}<0.05)$ when compared with the model group at 4 weeks. Furthermore, among YGD groups, the expression of CXCR4 and ERK1/2 increased and exhibited a significant difference $(\mathrm{P}<0.05)$. The relative mRNA expression levels of $\alpha$-SMA, NF- $\kappa B$ p 65 and $\beta$-catenin gradually decreased in the YGD groups in a time-dependent manner, almost reaching those of the control group at week 4, and revealing a significant decrease $(\mathrm{P}<0.05)$ compared with the model group at 4 weeks $(\mathrm{P}<0.05$; Fig. $4 \mathrm{C}$; Table IV).

\section{Discussion}

The mutagen DMN results in liver function disorders and cirrhosis. It is widely used in experimental animals, including mice, to create a low-mortality and reproducible model of liver cirrhosis $(8,9)$. DMN significantly induces the deposition of collagen fibers and the formation of fibrous septa, as reported by previous studies $(10,11)$, and as demonstrated by MT staining in the present study. Symptoms of typical liver cirrhosis appeared in the model group at week 4 and the $\alpha$-SMA level gradually increased, as demonstrated by IHC, western blotting and RT-qPCR. This revealed that the degree of liver fibrosis increased with the development of DMN-induced liver cirrhosis. In addition, the serum levels of ALT, AST, ALP, GGT, TBil and DBil significantly increased, whereas the serum Alb and CHE levels decreased following DMN administration. Furthermore, the infiltration of inflammatory cells, with destruction of the hepatic lobule, portal area structure and disordered arrangement of liver cells as revealed by HE staining suggested the presence of liver cell damage and defective liver function. This data suggested that the administration of DMN successfully induced liver cirrhosis in the model system of the current study.

HGF is a well-known angiogenic, anti-fibrotic and anti-inflammatory cytokine $(12,13)$. Certain studies (14-16) have reported that HGF induces MSCs to differentiate into hepatocytes in vitro and attenuate liver fibrosis in vivo. The anti-fibrotic effect of HGF may be achieved via inhibition of the proliferation and activation of hepatic stellate cells (HSCs), and attenuation of the expression of the fibrogenic cytokines transforming growth factor $\beta 1$ and platelet-derived growth factor-BB in the liver (17). The effectiveness and broad mode of action of this cytokine prompted the selection of HGF as a positive control to investigate the underlying mechanism of YGD in the reversal of liver cirrhosis. YGD is a traditional Chinese medicine in which Radix Rehmanniae is the primary 


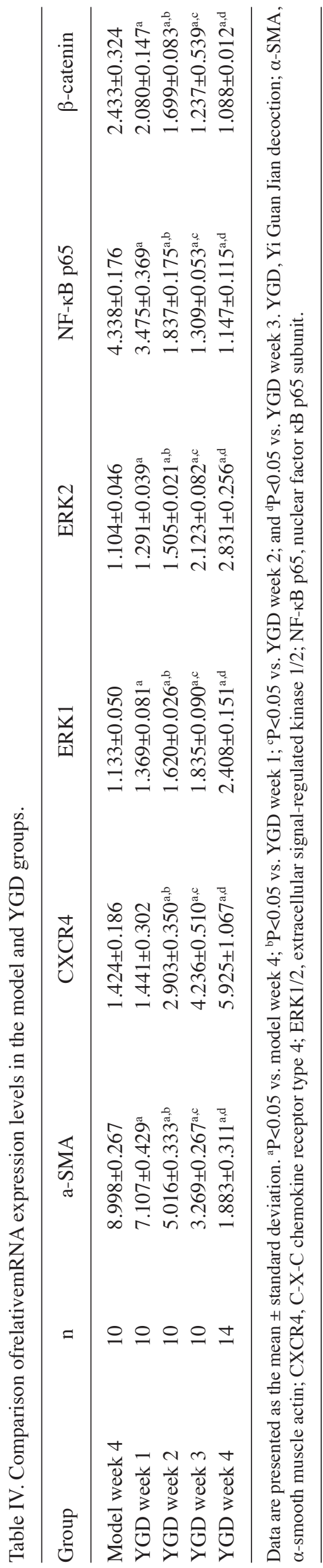

active substance, and has 'yin-blood-enriching' effects as well as providing nourishment to liver and kidney (18). The formula may cure the 'liver-kidney yin deficiency' (19) syndrome of chronic hepatitis and liver cirrhosis. In addition, it may induce the differentiation of rat BMSCs into HLCs (20). Following YGD or HGF treatment, the serum levels of ALT, AST, Alb, CHE, ALP, GGT, TBil and DBil demonstrated a marked improvement compared with the model group. Liver histology indicated that the necrotic degeneration of liver cells gradually decreased, regeneration of the hepatic cord disordered arrangement occurred and hyperplasia of fibrous tissue and fibrous septa gradually reduced. Elevation of $\alpha$-SMA mRNA and protein expression levels, which is a marker of HSC activation, were additionally inhibited. This data indicated that YGD achieved a similar effect to HGF on improvement of liver function and structure, and reversing DMN-induced liver cirrhosis. This may be mediated via inducing the differentiation of BMSCs into HLCs and inhibiting the activation of HSCs to reduce the expression of $\alpha$-SMA.

BMSCs are multipotent cells capable of differentiating into multiple lineages that contribute to tissue repair and regeneration, including adipocytes, osteoblasts, hepatocytes, nerve cells, chondroblasts and myocardial cells (21). A previous study has demonstrated that BMSCs may repair liver function, promote the regeneration of hepatocytes and reduce liver fibrosis via inhibiting HSC activation (22). The present study monitored the differentiation of BMSCs by analyzing the co-localization of CD90 with the hepatocyte marker Alb or the biliary epithelial cell marker CK18. In the DMN-induced cirrhotic liver, the co-expression of the two pairs of markers was significantly upregulated to a similar level, compared with the control mice, which indicated a balanced differentiation to the two epithelial cell subtypes. This differentiation, induced in response to injury, may be limited, and therefore not sufficient to reverse the damage induced by DMN. Following YGD treatment, the co-localization of CD90 with Alb and CK18 was increased, suggesting that YGD exerted a potent pro-differentiation effect on BMSCs, which may have reversed the DMN-induced liver cirrhosis.

The underlying mechanism of BMSC differentiation into hepatocytes and biliary epithelial cells remains to be elucidated; however, previous studies (23) suggest it is associated with multiple signaling pathways. The chemokine stromal cell-derived factor-1 (SDF-1) binds to CXCR4. Numerous studies have reported that SDF-1 is critical for stem/progenitor and mesenchymal cell chemotaxis and inflammatory cell homing to injured tissue via interaction with CXCR4 on the surface of these cells; CXCR4 maybe highly expressed in humans and mice following chemotaxis along an SDF-1 gradient $(24,25)$. This indicates that the level of CXCR4 expression may reflect the activation of the SDF-1/CXCR4 axis, which is associated with stem cells. The mitogen activated protein kinase (MAPK)/ERK1/2 signaling pathway is one of the downstream signaling pathways of SDF-1/CXCR4, which maybe activated by various growth factors and cytokines via phosphorylation and is involved in regulating the cell cycle and promoting cell proliferation and differentiation (26). In the present study, following administration of DMN, the mRNA and protein expression levels of CXCR4 
and ERK1/2 increased significantly; these levels increased further during YGD treatment, indicating that YGD induces the differentiation of BMSCs into HLCs by activating SDF-1/CXCR4 and directly phosphorylating the downstream effectors of the MAPK/ERK1/2 signaling pathway.

$N F-\kappa B$ is responsible for the rapid induction of various cytokines and adhesion molecules involved in immune and inflammatory responses, is the downstream substrate for numerous cytokine signaling pathways and is important in regulating the proliferation, survival and apoptosis of cells. Following cellular stimulation, inhibitor of $\kappa \mathrm{B} \alpha$ is phosphorylated and promotes the translocation of $\mathrm{NF}-\kappa \mathrm{B}$ to the nucleus where it binds to NF- $\kappa \mathrm{B}$ DNA binding sites and induces transcription. Previous studies have verified that $\mathrm{NF}-\kappa \mathrm{B}$ may participate in adjusting the activation of Kupffer cells and HSCs during liver fibrosis (27), which is regulated by the MAPK/ERK1/2 and Wnt/ $\beta$-catenin signaling pathways via the differentiation of BMSCs $(28,29)$. In the present study, the NF- $\mathrm{B}$ p65 mRNA and protein expression levels increased following DMN administration, and gradually decreased following YGD treatment; these results indicated that YGD induced differentiation of BMSCs to reverse liver fibrosis by inhibiting the activation of HSCs, leading to a decrease in NF- $\kappa \mathrm{B}$ p65 levels.

Furthermore, the present study observed that the mRNA and protein expression levels of $\beta$-catenin increased following DMN administration, and reduced following YGD treatment, which indicated that YGD inhibited $\beta$-catenin expression and thus induced the differentiation of BMSCs into HLCs. $\beta$-catenin is a multifunctional protein, which is important in the regulation of cell differentiation and proliferation. A previous study suggested that Wnt/ $\beta$-catenin signaling pathway downregulation may induce the differentiation of BMSCs into HLCs (30); however, in the context of the present study, this remains to be elucidated.

In conclusion, the results of the present study suggested that YGD reverses DMN-induced liver cirrhosis in mice, and improves liver function and structure. YGD may act via upregulation of the SDF-1/CXCR4 axis, which activates the MAPK/ERK1/2 signaling pathway, leading to NF- $\kappa \mathrm{B}$ p65 downregulation, thus inducing the differentiation of BMSCs into HLCs and inhibiting activation of HSCs.

Finally, there are remain several shortcomings in the present study that require further improvement. The model group at week 4 demonstrated more severe damage according to measurements of serum liver function, pathology and IHC, compared with the $\mathrm{NC}$ group at any time point, so it was selected for comparison with the YGD and HGF groups in Western blotting and RT-qPCR experiments, but not the NC group. This may be a limitation of this study, and future experiments are required in order to make the substantiate the conclusions drawn in this work.

\section{Acknowledgements}

The present study was supported by the National Natural Science Foundation of China (grant nos. 81273925 and 81673728) and the Scientific Research Foundation of Dalian (grant no. 2012E15SF143). The authors thank the Central Laboratory of the First Affiliated Hospital of Dalian Medical University for support with experimental techniques.

\section{References}

1. Wu XB and Tao R: Hepatocyte differentiation of mesenchymal stem cells. HepatobiliaryPancreat Dis Int 11: 360-371, 2012.

2. Pilat N, Unger L and Berlakovich GA: Implication for bone marrow stem cells in hepatocyte regeneration after orthotopic liver transplantation. Int J Hepatol 2013: 310612, 2013.

3. Wang L, Ping L, Yongping M, et al: Study on TCM recipe and syndrome of dimethylnitrosamine-induced hepatic fibrosis in rats. J Trad Chin Med 47: 929-932, 2006.

4. Min L, Zhiying $\mathrm{C}$ and Guobin W: Investigate Yiguanjian treatment the liver-kidney yin deficiency syndrome of posthepatitic cirrhosis. TCM Research 22: 3-4, 2009.

5. Ying Z and Ping Z: Effect of YGJ on proliferation and differenciation of hepatic oval cells in rat cirrhosis by DMN. Journal of Dalian Medical University 33: 11-16, 2011.

6. Jenkins SA, Crandison A, Baxter JN, Day DW, Taylor I and Shields R: A dimethyl nitrosamine-induced model of cirrhosis and portal hypertension in the rat. J Hepatol 1: 489-499, 1985.

7. Schmittgen TD, Zakrajsek BA, Mills AG, Gorn V, Singer MJ and Reed MW: Quantitative reverse transcription-polymerase chain reaction to study Mrna decay: Comparison of endpoint and real-timemethods. Anal Biochem 285: 194-204, 2000.

8. Jézéquel AM, Mancini R, Rinaldesi ML, Ballardini G, Fallani M, Bianchi F and Orlandi F: Dimethylnitrosamine-induced cirrhosis. Evidence for an immunological mechanism. J Hepatol 8: 42-52, 1989.

9. Kim KS, Yang HJ, Lee JY, Na YC, Kwon SY, Kim YC, Lee JH and Jang HJ: Effects of $\beta$-sitosterol derived from Artemisia capillaris on the activated human hepatic stellate cells and dimethylnitrisamine-induced mouse liver fibrosis. BMC Complement Altern Med 14: 363, 2014.

10. Nishibe Y, Kaneko H, Suzuki H, Abe T, Matsuura Y and Takaku H: Baculovirus-mediated interferon alleviates dimethyl nitrosamine-induced liver cirrhosis symptomsin a murine model. Gene Ther 15: 990-997, 2008.

11. Chen P, Li J, Huo Y, Lu J, Wan L, Yang Q, Huang J, Gan R and Guo C: Adenovirus-mediated expression of orphan nuclear receptor NR4A2 targeting hepatic stellate cell attenuates liver fibrosis in rats. Sci Rep 6: 33593, 2016.

12. Bell LN, Cai L, Johnstone BH, Traktuev DO, March KL and Considine RV: A central role for hepatocyte growth factor in adipose tissue angiogenesis. Am J Physiol Endocrinol Metab 294: E336-E344, 2008

13. Inagaki Y, Higashi K, Kushida M, Hong YY, Nakao S, Higashiyama R, Moro T, Itoh J, Mikami T, Kimura T, et al: Hepatocyte growth factor suppresses profibrogenic signal transduction via nuclear export of Smad3 with galectin-7. Gastroenterology 134: 1180-1190, 2008.

14. Oh SH, Miyazaki M, Kouchi H, Inoue Y, Sakaguchi M, Tsuji T, Shima N, Higashio K and Namba M: Hepatocyte growth factor induces differentiation of adult rat bone marrow cells into a hepatocyte lineage in vitro. Biochem Biophys Res Commun 279: 500-504, 2000.

15. OkumotoK, Saito T,Hattori E,Ito JI,Adachi T, Takeda T, SugaharaK, Watanabe H, Saito K, Togashi H and Kawata S: Diffferentiation of bone marrow cells into cells that express liver-specific genes in vitro: Implication of the Notch signals in differentiation. Biochem Biophys Res Commun 304: 691-695, 2003.

16. van de Kamp J, Jahnen-Dechent W, Rath B, Knuechel R and Neuss S: Hepatocyte growth factor-loaded biomaterials for mesenchymal stem cell recruitment. Stem Cells Int 2013: $892065,2013$.

17. Kim WH, Matsumoto K, Bessho K and Nakamura T: Growth inhibition and apoptosis in liver myofibroblasts promoted by hepatocyte growth factor leads to resolution from liver cirrhosis. Am J Pathol 166: 1017-1028, 2005.

18. Tian DD, Wang W, Wang HN, Sze SC and Zhang ZJ: Pharmacokinetic evaluation of clozapine in concomitant use of Radix Rehmanniae, fructus schisandrae, radix bupleuri, or fructus gardeniae in rats. Molecules 21: E696, 2016.

19. Lu YY, Zhao Y, Song YN, Dong S, Wei B, Chen QL, Hu YY and Su SB: Serum cytokine profiling analysis for zheng differentiation in chronic hepatitis B. Chin Med 10: 24, 2015.

20. Ping J, Chen HY, Yang Z, Yang C and Xu LM: Effect of yiguan decoction on differentiation of bone marrow mesenchymal stem cells into hepatocyte-like cells: An experimental research. Zhongguo Zhong Xi Yi Jie He Za Zhi 34: 348-354, 2014 (In Chinese). 
21. Anzalone R, Lo Iacono M, Corrao S, Magno F, Loria T, Cappello F, Zummo G, Farina F and La Rocca G: New energing potentials for human Wharton's jelly mesenchymal stem cells: Immunological features and hepatocyte-like differentiative capacity. Stem Cells Dev 19: 423-438, 2010.

22. Haridass D, Narain N and Ott M: Hepatocyte transplantation: Waiting for stem cells. CurrOpin Organ Transplant 13: 627-632, 2008.

23. Facciorusso A, Antonino M, Del Prete V, Neve V, Scavo MP and Barone M: Are hematopoietic stem cells involved in hepatocarcinogenesis? Hepatobiliary Surg Nutr 3: 199-206, 2014.

24. Kucia M, Ratajczak J, Reca R, Janowska-Wieczorek A and Ratajczak MZ: Tissue-specific muscle, neural and liver stem/progenitor cells reside in the bone marrow, respond to an SDF-1 gradient and are mobilized into peripheral blood during stress and tissue injury. Blood Cells Mol Dis 32: 52-57, 2004.

25. Sun L, Fan X, Zhang L, Shi G, Aili M, Lu X, Jiang T and Zhang Y: Bone mesenchymal cell transplantation via four routes for the treatment of acute liver failure in rats. Int J Mol Med 34: 987-996, 2014

26. Delgado-Martín C, Escribano C, Pablos JL, Riol-Blanco L and Rodríguez-Fernández JL: Chemokine CXCL12 uses CXCR4 and a signaling core formed by bifunctional AKT, extracellular signal-regulated kinase (ERK) $1 / 2$ and mammalian target of rapamycin complex $1(\mathrm{~m}$ TORC1) proteins to control chemotaxis and survival simultaneously in mature dendritic cells. J Biol Chem 286: 37222-37236, 2011.
27. Luedde $\mathrm{T}$ and Schwabe RF: NF- $\mathrm{BB}$ in the liver-linking injury, fibrosis and hepatocellular carcinoma. Nat Rev Gastroenterol Hepatol 8: 108-118, 2011.

28. Chang J, Liu F, Lee M, Wu B, Ting K, Zara JN, Soo C, Al Hezaimi K, Zou W, Chen X, et al: NF- $\kappa$ B inhibits osteogenic differentiation of mesenchymal stem cells by promoting $\beta$-catenin degradation. Proc Natl Acad Sci USA 110: 9469-9474, 2013.

29. Pandey AC, Semon JA, Kaushal D, O'Sullivan RP, Glowacki J, Gimble JM and Bunnell BA: MicroRNA profiling reveals age-dependent differential expression of nuclear factor $\kappa \mathrm{B}$ and mitogen-activated protein kinase in adipose and bone marrow-derived human mesenchymal stem cells. Stem Cell Res Ther 2: 49, 2011.

30. Ke Z, Zhou F, Wang L, Chen S, Liu F, Fan X, Tang F, Liu D and Zhao G: Down-regulation of Wnt signaling could promote bone marrow-derived mesenchymal stem cells to differentiate into hepatocytes. Biochem Biophys Res Commun 367: 342-348, 2008. 